Methods Utilising our human 3-D vaginal EC model, that more accurately recapitulates in vivo human vaginal tissue, we tested the hypothesis that IL-36r induction in the vaginal epithelium is microbe-dependent by testing a panel of STI microbes and microbial products. To further investigate the induction and regulation of IL-36 $\gamma, 3-\mathrm{D}$ vaginal EC were treated with poly (I:C), flagellin or FSL-1 for $24 \mathrm{~h}$. Human 3-D cells were analysed by real-time qPCR analysis. Cell pellets and culture supernatants were also collected and analysed by IL-36 $\gamma$ ELISA, Western blot and cytometric bead array.

Results Following exposure to STI pathogens (herpes simplex virus and bacterial vaginosis (BV)-associated bacteria) and specific microbial products, IL-36 $\gamma$ expression was significantly increased relative to untreated and Lactobacilli spp. bacteria in the vaginal EC model. All microbial products tested significantly $(p<0.05)$ induced expression of IL-36 $\gamma$ in a dose- and TLRdependent manner. Treatment with IL-36 $\gamma$ significantly $(\mathrm{p}<$ 0.05 ) induced proinflammatory cytokines and antimicrobial pep-

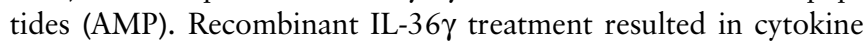
and AMP production, thereby promoting inflammation in the local microenvironment.

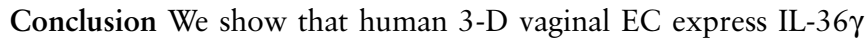
and this cytokine is elicited in a microbe-dependent manner at this mucosal site. Furthermore, we demonstrate that IL-36r is an important driver for epithelial activation and inflammation following infection with STI-related pathogens and BV-associated bacteria, as such this novel cytokine may play an important role in host defense in the vaginal epithelium.

Disclosure of interest statement No pharmaceutical grants were received in the development of this study.

\section{P06.13 INFLAMMATORY CYTOKINE BIOMARKERS IDENTIFY WOMEN WITH ASYMPTOMATIC GENITAL INFECTIONS THAT INCREASE THE RISK OF HIV INFECTION}

1,2L Masson* ${ }^{3,4}{ }^{3}$ Deese, ${ }^{5} \mathrm{~KB}$ Arnold, ${ }^{6} \mathrm{~F}$ Little, ${ }^{2,7} \mathrm{~K}$ Mlisana, ${ }^{8,9,10} \mathrm{DA}$ Lewis, ${ }^{11} \mathrm{~L}$ Van Damme, ${ }^{12} \mathrm{~T}$ Crucitti, ${ }^{12} \mathrm{~S}$ Abdellati, ${ }^{10} \mathrm{~N}$ Mkhize, ${ }^{1} \mathrm{H}$ Gamieldien, ${ }^{2} \mathrm{~S}$ Ngcapu, ${ }^{5} \mathrm{DA}$ Lauffenburger, ${ }^{2,13} \mathrm{Q}$ Abdool Karim, ${ }^{2,13} \mathrm{SS}$ Abdool Karim, ${ }^{1,2,14} \mathrm{JS}$ Passmore. ${ }^{1}$ Institute of Infectious Diseases and Molecular Medicine, University of Cape Town Medical School, Cape Town, South Africa; ${ }^{2}$ Centre for the AIDS Programme of Research in South Africa, University of KwaZulu Natal, Durban, South Africa; ${ }^{3} \mathrm{FHI}$ 360, Durham, NC, USA; ${ }^{4}$ Department of Epidemiology, Gillings School of Global Public Health, The University of North Carolina at Chapel Hill, Chapel Hill, NC, USA; ${ }^{5}$ Department of Biological Engineering, Massachusetts Institute of Technology, Cambridge, Massachusetts, USA; ${ }^{6}$ Department of Statistical Sciences, University of Cape Town, Cape Town 7701, South Africa; ${ }^{7}$ School of Laboratory Medicine and Medical Sciences, University of KwaZulu-Natal; ${ }^{8}$ Western Sydney Sexual Health Centre, Parramatta, Australia; ${ }^{9}$ Centre for Infectious Diseases and Microbiology \& Marie Bashir Institute for Infectious Diseases and Biosecurity, Westmead Clinical School, University of Sydney, Sydney, Australia; ${ }^{10}$ National Institute for Communicable Diseases, Sandringham, Johannesburg, South Africa; ${ }^{11}$ The Bill \& Melinda Gates Foundation, Seattle, Washington, USA (Formerly of FHI 360); ${ }^{12}$ Institute of Tropical Medicine, Antwerp, Belgium; ${ }^{13}$ Columbia University, New York, New York, USA; ${ }^{14}$ National Health Laboratory Services, South Africa

\subsection{6/sextrans-2015-052270.314}

Introduction Untreated sexually transmitted infections (STIs) and bacterial vaginosis (BV) cause genital inflammation and increased risk of HIV infection. WHO-recommended syndromic STI and BV management is limited as large numbers of women with asymptomatic infections go untreated. The purpose of this study was to evaluate genital cytokine profiles as a biomarker to identify women with asymptomatic, treatable infections.

Methods Luminex was used to measure the concentrations of 42 cytokines in cervicovaginal lavages (CVL) from 227 HIV- uninfected women from Durban, South Africa, and nine cytokines in endocervical swabs from 264 women from Bondo, Kenya and Pretoria, South Africa. Women were screened for BV and treatable STIs (Chlamydia trachomatis, Neisseria gonorrhoeae, Trichomonas vaginalis, Mycoplasma genitalium) using microscopy and molecular assays. Nonparametric receiver operating characteristic curves and logistic regression were used to identify cytokine profiles associated with STIs/BV.

Results In women from Durban, concomitant increased IL- $1 \alpha$ and IL-1 $\beta$ and decreased IP-10 concentrations in CVLs predicted the presence of a treatable genital condition, correctly classifying $76 \%$ of women (sensitivity $72 \%$, specificity $81 \%$, PPV $86 \%$ and NPV 64\%). In a separate validation cohort of women from Bondo and Pretoria, IL- $1 \alpha$, IL-1 $\beta$ and IP-10 concentrations in endocervical swabs correctly classified $72 \%$ of the participants according to STI/BV status. This approach performed substantially better than clinical signs in both cohorts from Durban (sensitivity 19\%, specificity 92\%, PPV 79\% and NPV 40\%) and Pretoria and Bondo (sensitivity 29\%, specificity 78\%, PPV 68\%, NPV 39\%).

Conclusion Across two cohorts of women residing in different regions in sub-Saharan Africa, genital IL- $1 \alpha$, IL- $1 \beta$ and IP-10 together was the best immunological predictor of the presence of an STI or BV. Supplementing syndromic management with point-of-care assessment of biomarkers of genital inflammation may improve STI/BV management for women, enabling more effective treatment of asymptomatic infections and potentially reducing their risk of HIV infection.

Disclosure of interest statement This work was supported by a Strategic Health Innovation Partnerships (SHIP) grant from the South African Medical Research Council and grants from the Poliomyelitis Research Foundation (PRF) of South Africa and European and Developing Countries Clinical Trials Partnership (EDCTP). The cohorts were supported by grants from the Comprehensive International Program of Research on AIDS (CIPRA) of the Division of AIDS (DAIDS); National Institute of Allergy and infectious Disease (NIAID); National Institutes of Health (NIH) and US Department of Health and Human Services (DHHS) [grant number U19 AI51794]. FEM-PrEP was conducted under two grants funded by the United States Agency for International Development (USAID): the Contraceptive and Reproductive Health Technologies and Research Utilisation Program (GPO-A-00-05-00022-00), and the Preventive Technologies Agreement (GHO-A-00-09-00016-00). Early support was also provided by the Bill and Melinda Gates Foundation. Gilead Sciences, Inc. donated Truvada ${ }^{\circledR}$ and placebo. LM was supported by the PRF; South African Medical Research Council (MRC); the Carnegie Corporation; the National Research Foundation (NRF) of South Africa and the UCT Clinical Infectious Diseases Research Initiative/Wellcome Trust. No pharmaceutical grants were received in the development of this study.

\section{P06.14 THE EFFECT OF SEXUAL INTERCOURSE ON VAGINAL COLONISATION WITH CANDIDA}

${ }^{1,2} \mathrm{CJ}$ Watson*, ${ }^{3} \mathrm{CK}$ Fairley, ${ }^{4} \mathrm{SM}$ Garland, ${ }^{5} \mathrm{~S}$ Myers, ${ }^{1} \mathrm{M}$ Pirotta. ${ }^{1}$ Department of General Practice, University of Melbourne, Melbourne; 'Women's Health Clinics, Royal Women's Hospital, Melbourne; ${ }^{3}$ School of Population Health, University of Melbourne; Melbourne Sexual Health Centre, Alfred Health, Melbourne; ${ }^{4}$ Department Microbiology Infectious Diseases, Royal Women's Hospital; Department of Obstetrics and Gynaecology University of Melbourne, Melbourne; ${ }^{5}$ NatMed-Research, Southern Cross University, Lismore

10.1136/sextrans-2015-052270.315 
Introduction Recurrent vulvovaginal candidiasis (RVVC) results in significant physical, financial and psychological sequelae for women, and many women report that VVC affects their intimate relationships. The aetiology of RVVC remains uncertain, and some studies suggest sexual intercourse may be responsible for transmission of Candida species. No publications have documented the affect of sexual intercourse on vaginal candida colonisation.

Methods Fifty nine participants who were culture positive for Candida spp. at screening took part in a randomised controlled trial investigating the effect of oral garlic and placebo on vaginal candidal colonisation. Participants self-collected daily vaginal swabs during the two weeks before menstruation. They kept a daily diary and recorded incidence of sexual intercourse and abnormal vaginal symptoms. Swabs were analysed for quantitative colony counts of candida before and after sexual intercourse.

Results There were 149 episodes of sexual intercourse in participants reporting sexual activity $(n=38)$ over the two week study period. Colonisation levels rose the day following sexual intercourse in 51 episodes, and fell in 56 episodes. In 42 episodes of sexual intercourse, the levels remained the same or women were culture negative on the day following and two days following sexual intercourse. On fifty occasions women had symptoms (itch, abnormal vaginal discharge) on the day of sexual intercourse, and 41 women reported abnormal symptoms two days after sexual intercourse. In 75 episodes, there were no abnormal symptoms the day of, or the day following sexual intercourse.

Conclusion In this study, sexual intercourse, colonisation levels and abnormal vaginal symptoms appeared to be unrelated. Further investigation is recommended into dyspareunia and abnormal vaginal symptoms following sexual intercourse experienced by women with RVVC.

Disclosure of interest statement No pharmaceutical grants were received in the development of this study.

\section{P06.15 THE STABILITY OF THE VAGINAL MICROBIOME IN RELATION TO NEW SEXUAL EXPERIENCES}

1,2) Twin, ${ }^{3,4,5} \mathrm{CS}$ Bradshaw, ${ }^{3}$ LA Vodstrcil, ${ }^{1,2,4} \mathrm{SM}$ Garland, ${ }^{3,5} \mathrm{CK}$ Fairley, ${ }^{3} \mathrm{G}$ Fehler, ${ }^{4} \mathrm{JA}$ Hocking, ${ }^{5} \mathrm{M}$ Law, ${ }^{3} \mathrm{KA}$ Fethers, ${ }^{1,2,4} \mathrm{SN}$ Tabrizii*. ${ }^{1}$ Royal Women's Hospital; ${ }^{2}$ Murdoch Children's Research Institute; ${ }^{3}$ Melbourne Sexual Health Centre; ${ }^{4}$ University of Melbourne; ${ }^{5}$ Monash University, Kirby Institute

\subsection{6/sextrans-2015-052270.316}

Introduction The vaginal microbiome in healthy asymptomatic women can vary greatly, though in most is typically dominated by lactic acid producing Lactobacillus spp. which convey protection against pathogens. Few studies have examined genital bacterial communities in sexually-inexperienced women over time and particularly changes occurring upon initiation of new sexual experiences.

Methods Overall192 samples from 45 healthy women were selected as a subset of a study investigating the sexual health of 17-21 year old university students. Women who were selected included those with no previous sexual experience $(\mathrm{n}=17)$, women experienced in non-coital sexual activities only $(\mathrm{n}=15)$ and women who had engaged in penile-vaginal sex $(n=13)$. The selected participants provided self-collected vaginal swab samples every 3 months for 12 months. Bacterial communities were analysed using Roche 454 amplicon sequencing with PCR primers targeting the V3/4 variable region of the $16 \mathrm{~S}$ rRNA gene.
Results Overall, healthy young women had differing vaginal community states. Onset of non-coital and coital sexual activities does not exert a significant effect on the composition of vaginal bacterial communities. The vaginal community-state with the greatest stability over time consisted of states predominated by L. crispatus and L. iners with median Bray-curtis dissimilarity values of 12.4 and 17.6 respectively ( $\mathrm{p}=0.005$ and 0.024 ). Vaginal microbiomes dominated by other Lactobacillus spp. and non-Lactobacillus spp. gave rise to the most variability over time (dissimilarity values of 41.1 and 66.8). Non-coital and coital sexual activity within this subset of participants did not have any significant effect upon the stability of vaginal bacterial communities $(p=0.3714)$.

Conclusion L. crispatus and L. iners are most commonly found to dominate sexually inexperienced women and convey the most stable environment over time. The initiation of new sexual activities does not appear to have any persistent effect on the vaginal microbiome of young women.

Disclosure of interest statement None to declare.

\section{P07 - STI/HIV diagnosis}

\section{P07.01 MYCOPLASMA GENITALIUM TESTING PATTERN AND INFECTION RATES OVER A SIX-YEAR PERIOD IN MELBOURNE, AUSTRALIA}

${ }^{1,2,3} \mathrm{SN}$ Tabrizi* ${ }^{2}{ }^{2} \mathrm{AM}$ Costa, ${ }^{2} \mathrm{~A}$ Marceglia, ${ }^{1,2} \mathrm{DA}$ Machalek, ${ }^{4} \mathrm{CS}$ Bradshaw, ${ }^{4} \mathrm{M}$ Chen, ${ }^{4} \mathrm{CK}$ Fairley, ${ }^{1,2,3} \mathrm{SM}$ Garland. 'Murdoch Childrens Research Institute; ${ }^{2}$ The Royal Children's and the Royal Women's Hospitals; ${ }^{3}$ University of Melbourne; ${ }^{4}$ Melbourne Sexual Health Centre

\subsection{6/sextrans-2015-052270.317}

Introduction Mycoplasma genitalium ( $\mathrm{Mg})$ is an emerging sexually transmitted pathogen with a strong association with urethritis, cervicitis and pelvic inflammatory disease. Detection of this bacterium using molecular assays has been limited due to lack of readily available commercial assays. However, in house $16 \mathrm{~S}$ rRNA gene qPCR assays have been in use at the laboratory located at the Royal Women's Hospital, in Melbourne Australia for detection of $\mathrm{Mg}$ since 2009. The aim of this study was to analyse $\mathrm{Mg}$ testing patterns and infection rates over this 6 year period.

Methods We analysed overall detection rates and site-specific positivity in clinical specimens received for testing for $\mathrm{Mg}$ between 1 January 2009 and 31 December 2014 from clinics at the Royal Women's Hospital and Melbourne Sexual Health Centre.

Results A total of 46,112 specimens were tested for $\mathrm{Mg}$; 2,853 $(6.2 \%)$ samples were tested in 2009 with an increasing trend to $13,133(28.5 \%)$ in 2014 ( $\mathrm{p}$-trend <0.001). In total $54.7 \%$ were urine samples, $37.7 \%$ vaginal/cervical swabs and $7.6 \%$ were anal, urethral or from other non-specified sites. Overall positivity across all samples was 4.5\% (95\% CI: 4.3-4.7) without any significant change per annum ( $\mathrm{p}$-trend $=0.206$ ). Overall, $\mathrm{Mg}$ detection rate was highest in urethral $(9.0 \%, 95 \%$ CI: $6.7-11.7)$ and anal swabs $(8.8 \%, 95 \%$ CI $6.8-11.1)$ followed by urine (5.8\%, 95\% CI: 5.5-6.7) and cervical/vaginal samples (2.6\%, 95\% CI: $2.4-2.8$ ) ( $\mathrm{p}<0.001)$. A significant increase in positivity was observed in anal swabs, from $2.5 \%$ in 2009 to $12.7 \%$ in 2014 (p-trend $=0.005)$.

Conclusion Increased testing for $\mathrm{Mg}$ by $\mathrm{qPCR}$ has resulted in detection and treatment of over 2000 infections since 2009 in 